\title{
Computational investigation of the lubrication behaviors of dioxides and disulfides of molybdenum and tungsten in vacuum
}

\author{
Jingyan NIAN ${ }^{1}$, Liwei CHEN ${ }^{2}$, Zhiguang GUO ${ }^{1,2, *}$, Weimin LIU $^{1}$ \\ ${ }^{1}$ State Key Laboratory of Solid Lubrication, Lanzhou Institute of Chemical Physics, Chinese Academy of Sciences, Lanzhou 730000, China \\ ${ }^{2}$ Hubei Collaborative Innovation Centre for Advanced Organic Chemical Materials and Ministry of Education Key Laboratory for the \\ Green Preparation and Application of Functional Materials, Hubei University, Wuhan 430062, China
}

Received: 18 September 2016 / Revised: 02 November 2016 / Accepted: 03 November 2016

(C) The author(s) 2016. This article is published with open access at Springerlink.com

\begin{abstract}
Lamellar compounds such as the disulfides of molybdenum and tungsten are widely used as additives in lubricant oils or as solid lubricants in aerospace industries. The dioxides of these two transition metals have identical microstructures with those of the disulfides. The differences in the lubrication behaviors of disulfide and dioxides were investigated theoretically. Tungsten dioxide and molybdenum dioxide exhibit higher bond strengths at the interface and lower interlayer interactions than those of the disulfides which indicates their superlubricity. Furthermore, the topography of the electron density of the single layer nanostructure determined their sliding potential barrier; the dioxides showed a weaker electronic cloud distribution between the two neighboring oxygen atoms, which facilitated the oxygen atoms of the counterpart to go through. For commensurate friction, the dioxides exhibited nearly the same value of friction work, and same was the case for the disulfides. The lower positive value of friction work for the dioxides confirmed their improved lubricity than the disulfides and the higher mechanical strength of the bulk dioxides demonstrated that they are excellent solid lubricants in vacuum.
\end{abstract}

Keywords: solid lubricant; superlubricity; first-principles; molecular dynamics; disulfides; dioxides

\section{Introduction}

Molybdenum disulfide $\left(\mathrm{MoS}_{2}\right)$, a well-known lamellar compound has been widely used in the aerospace industry and as an additive in lubricant due to its low friction coefficient [1-3]. Stefanov et al. [4] studied the lubrication mechanism of $\mathrm{MoS}_{2}$ nanotubes at the atomic scale using molecular dynamics and found that the lubrication process at high loads is not determined by the ball-bearing effect; the nanotube is compressed to form a nanosheet resulting in excellent lubrication performance. Onodera et al. [5] studied the friction process between the interlayers of $\mathrm{MoS}_{2}$ nanosheet using a hybrid quantum chemical/classical molecular dynamics method. The results indicated that the strong Coulombic force of repulsion between the interlayers of the nanosheets leads to the low friction; if the $\mathrm{MoS}_{2}$ nanosheets are adsorbed on a substrate with electrondonating property, the friction will be further reduced due to the larger Coulombic force of repulsion. The dynamic friction of two-layer $\mathrm{MoS}_{2}$ nanosheets at the atomic level was also theoretically investigated by the molecular dynamics method by Onodera et al. [6]. It was demonstrated that incommensurate structures showed extremely low friction due to the cancellation of the atomic forces in the sliding direction. On the other hand, in commensurate structures, the atomic forces were formed by the approaching of atoms in sliding part with the atoms in its counterpart, and all the atomic forces acted in the same direction, leading to the accumulation of lateral forces and higher friction. Wang et al. [7] investigated the friction property of in-plane stretch and compression of $\mathrm{MoS}_{2}$ sheets and reported that the in-plane compression can also reduce

* Corresponding author: Zhiguang GUO, E-mail: zguo@licp.cas.cn 
the friction due to the decrease in the potential energy of sliding. Levita et al. [8] studied the influence of load and interlayer orientation on the friction of $2 \mathrm{H} \mathrm{MoS}$ using the $a b$ initio method and the results indicated that the electrostatic interaction progressively affects the sliding motion at higher loads.

In addition to $\mathrm{MoS}_{2}$, tungsten disulfide $\left(\mathrm{WS}_{2}\right)$ is also an important solid lubrication material that has been used for various tribological applications. However, theoretical studies on the friction behavior of $\mathrm{WS}_{2}$ have been rarely carried out to quantitatively reveal the differences in the lubrication behaviors of $\mathrm{MoS}_{2}$ and $\mathrm{WS}_{2}$. This motivated us to study the lubrication behavior of $\mathrm{WS}_{2}$. Furthermore, the superlubricity of tungsten dioxide $\left(\mathrm{WO}_{2}\right)$ was demonstrated by Cahangirov et al. [9] using the "Prandtl-Tomlinson model" $[10,11]$. Therefore, it would be interesting to study the lubrication property of molybdenum dioxide.

In the present work, the differences in the lubrication behaviors of $\mathrm{MoS}_{2}, \mathrm{WS}_{2}, \mathrm{MoO}_{2}$, and $\mathrm{WO}_{2}$ solid lubricants were investigated using the first principles theory and molecular dynamic simulations.

\section{Computational details}

All the solid lubricant materials mentioned above consist of hexagonal unit cells, formed by stacking $\mathrm{X}-\mathrm{M}-\mathrm{X}(\mathrm{M}=\mathrm{Mo}, \mathrm{W} ; \mathrm{X}=\mathrm{S}, \mathrm{O})$ trilayers; hexagonal unit cells are weakly bonded to each other by the van der Waals forces. It is to be noted that the stable existence of these single layer solid materials has been demonstrated through optimization of the geometries and phonon dispersion calculations based on firstprinciples [12-14].

\subsection{Intrinsic properties of single layer nanostructures}

Considering single layer nanostructures as the research models, all the density functional theory plane-wave pseudopotential calculations were carried out using CASTEP $[15,16]$ which is a state-of-the-art quantum mechanics-based program. The intrinsic properties such as electronic structures and density of states were determined by LDA-CA-PZ (Ceperly and Alder's local density approximation $[17,18])$ methods. The kinetic energy cutoff for the plane-wave basis set was $300 \mathrm{eV}$, the convergence for energy was chosen as $5.0 \times 10^{-6} \mathrm{eV}$ between two steps, the maximum force allowed on each atom was less than $10^{-2} \mathrm{eV} / \AA$, the maximum stress and the displacement were 0.02 $\mathrm{GPa}$ and $5.0 \times 10^{-7} \mathrm{~nm}$ respectively, the Brillouin zone was sampled by $(12 \times 12 \times 2)$, and the ionic species were described by ultrasoft pseudopotentials.

\subsection{Interlayer sliding interaction}

The classic first-principle theory of atomic-scale friction proposed by Zhong and Tomanek [19] was used in the present study. Taking $\mathrm{MoO}_{2}(2 \times 2$ supercells $)$ for instance, the top-view of the calculation scheme is shown in Fig. 1. Ortmann, Bechstedt, and Schmid semiempirical dispersion-correction $[20,21]$ was added due to the van der Waals force between the interlayers. A vacuum region of $1.5 \mathrm{~nm}$ thickness was added in the [001] direction in order to avoid interaction between two single layers.

The interlayer interaction energies of two atomicscale slabs were calculated by Eq. (1).

$$
E_{\text {adsorption }}=E_{\text {Total }}-E_{\text {Slab1 }}-E_{\text {Slab2 }}
$$

$E_{\text {adsorption }}$ and $E_{\text {total }}$ represent the interaction energy and total energy of two $\mathrm{MoO}_{2}$ slabs $(2 \times 2$ supercells $)$ at different relative positions respectively. $E_{\text {slab1 }}$ and $E_{\text {slab2 }}$ are the energies of two slabs $(2 \times 2$ supercells $)$.

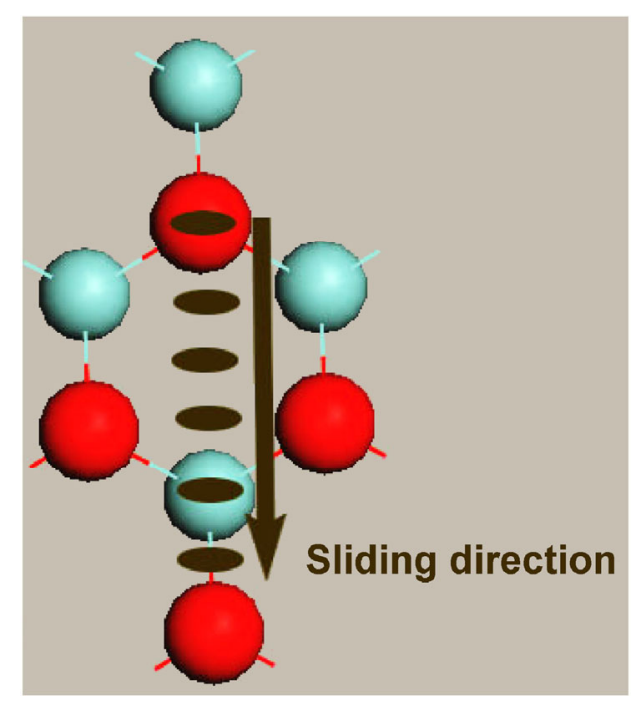

Fig. 1 Schematic diagram (top view) showing the relative sliding between two $\mathrm{MoO}_{2}(2 \times 2 \times 1)$ nanostructures. The ellipses indicate the relative positions and the arrow indicates the sliding direction. The red spheres represent the oxygen atoms and the light blue spheres represent the transition metal atoms. 
Considering the direction of the arrow shown in Fig. 1 as the sliding direction, $x$, the position-dependent parts of the potential energy, $V$ can be expressed as shown in Eq. (2).

$$
\begin{aligned}
V\left(x, f_{\text {ext }}\right)= & E_{\text {adsorption }}\left(x, z\left(x, f_{\text {ext }}\right)\right) \\
& +f_{\text {ext }} z\left(x, f_{\text {ext }}\right)-V_{0}\left(f_{\text {ext }}\right)
\end{aligned}
$$

Here, $V_{0}$ is the minimum potential energy corresponding to the $\mathrm{O}$ atom located at the hollow site relative to the other slab. In the case of atomic stick-slip motion, the conservative force results in an increase in the potential energy along the sliding path. This process is described by Eq. (3).

$$
\Delta V_{\text {max }}\left(f_{\text {ext }}\right)=V_{\text {max }}\left(f_{\text {ext }}\right)-V_{\text {min }}\left(f_{\text {ext }}\right)
$$

Therefore, the lateral position-dependent friction force, $f_{x}$ can be given by Eq. (4),

$$
\langle F\rangle=\frac{\Delta V_{\max }}{\Delta x}
$$

From Eq. (4), it is clear that the lubrication performance is associated with the potential barriers in the sliding path with minimum energy. The lower the value of the potential barrier, the better is the lubrication performance.

$$
f_{\text {ext }}=-\partial E_{\text {interaction }}(z) / \partial z
$$

According to Eq. (5), decreasing the interlayer distance is similar to applying a normal load. In the present system, the interlayer distance was decreased to $0.5 \AA$. It is to be noted that, the contour plots of the potential energy surfaces as functions of the different relative lateral positions of the two layers were constructed using the spline interpolation method [8].

\subsection{Mechanical properties of bulk materials}

The mechanical properties such as bulk modulus, Young's modulus, shear modulus, compressibility and in-plane Poisson's ratio of the bulk solid lubricant materials were also calculated using the first-principles theory. The shear modulus, $G$ can be calculated using Eq. (6) [22],

$$
G=\frac{1}{30}\left(C_{11}+C_{12}+2 C_{33}-4 C_{13}+12 C_{44}+12 C_{66}\right)
$$

Here, $C_{i j}$ and $C_{i i}$ are the elastic constants, $i$ and $j$ indicate the subscripts.

\subsection{Molecular dynamics simulation of commensurate sliding}

It is well known that commensurate surfaces usually appear in most true friction course, and thus molecular dynamic simulations were carried out by using Forcite modulus in Material Studio 6.0, Accelrys Software Inc. The commensurate friction models are shown in Fig. 2. The system temperature was set to $1 \mathrm{~K}\left(-272.15^{\circ} \mathrm{C}\right)$. The universal force field [23] which is a purely diagonal and harmonic force field was assigned to the transition metals and the light elements. The bond stretching was described by a harmonic term, the angle bending by a three-term Fourier cosine expansion, and the torsion and the inversion were described by Fourier cosine expansion terms. The van der Waals interactions were described by the LennardJones potential and the electrostatic interactions were described by atomic monopoles and a screened Coulombic term. The sliding velocity, $v$, was $0.05 \AA / p s$, and a complete stick-slip cycle was taken as the sliding distance. The positive friction work, $W_{\text {friction }}$ of the stick stage was used to evaluate the friction performance and calculated using Eq. (7).

$$
W_{\text {friction }}=\int_{0}^{t} F \cdot v \mathrm{~d} t
$$

The value of $F$ is obtained by summing the lateral forces acting on the atoms at the interface.

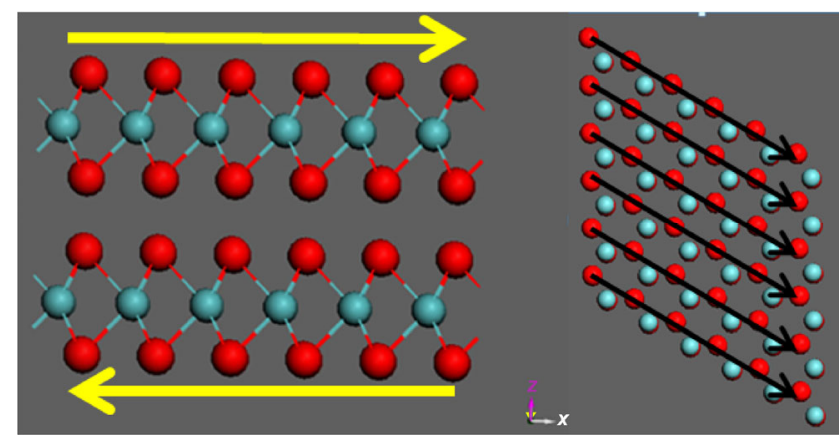

Fig. 2 Schematic showing the relative sliding between two single layer $\mathrm{MoO}_{2}(6 \times 6 \times 1)$ nanostructures; arrows show the sliding directions. The red spheres represent the oxygen atoms and the light blue spheres represent the transition metal atoms. 


\section{Results and discussion}

First, the intrinsic properties of the single layer dioxides and disulfides of molybdenum and tungsten are considered, which are summarized in Table 1 . The more charged light atoms at the interface correspond to a shorter interlayer distance in the commensurate condition, which indicates that the equilibrium interlayer distance is not determined by the electrostatic repulsion. In order to study the dry friction course of the solid lubricant materials at the atomic scale, it is necessary to investigate the intrinsic stiffness and the interlayer interaction. The intrinsic stiffness of a material can be evaluated from the bond stiffness at the interface. The density of states of the single layer nanostructures are shown in Fig. 3, which shows that the valence bands of all the nanostructures are composed of hybrids of $s, p$, and d orbitals. In addition, the degree of overlap of the $\mathrm{p}-\mathrm{d}$ orbitals between the transition metal and the light atoms is stronger than

Table 1 Intrinsic property of the single layer dioxides and disulfides of molybdenum and tungsten. The interlayer distance here is the equilibrium interlayer distance between the bottom atoms of the top layer and the top atoms of the bottom layer in a commensurate condition.

\begin{tabular}{ccccccc}
\hline System & Lattice constants $(\AA)$ & $d_{x-x}(\AA)$ & Bond length $(\AA)$ & Bond population & Hirshfeld charge $\begin{array}{c}\text { Interlayer } \\
\text { distance }(\AA)\end{array}$ \\
\hline $\mathrm{MoO}_{2}$ & $a=b=2.82$ & $d_{\mathrm{O}-\mathrm{O}}=2.42$ & 2.04 & 0.75 & O: $-0.25 ;$ Mo: 0.48 \\
$\mathrm{MoS}_{2}$ & $a=b=3.18$ & $d_{\mathrm{S}-\mathrm{S}}=3.11$ & 2.41 & 0.97 & $\mathrm{~S}:-0.11 ; \mathrm{Mo}: 0.48$ \\
$\mathrm{WO}_{2}$ & $a=b=2.84$ & $d_{\mathrm{O}-\mathrm{O}}=2.45$ & 2.05 & 0.83 & O: $-0.20 ; \mathrm{W}: 0.40$ \\
$\mathrm{WS}_{2}$ & $a=b=3.19$ & $d_{\mathrm{S}-\mathrm{S}}=3.13$ & 2.42 & 1.08 & $\mathrm{~S}:-0.06 ; \mathrm{W}: 0.12$ & 3.46 \\
\hline
\end{tabular}
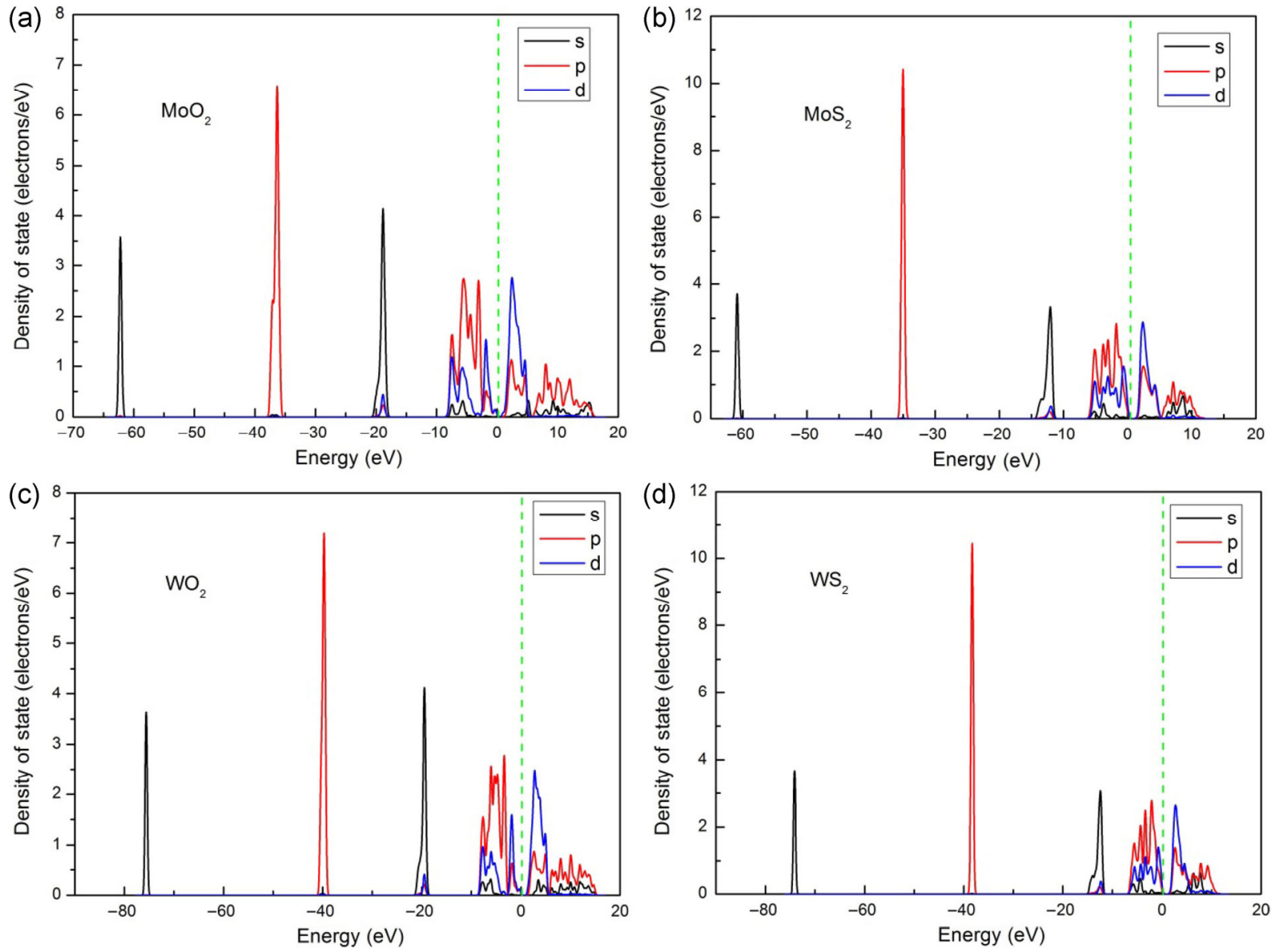

Fig. 3 Density of states distribution of single layer nanostructures of $\mathrm{MoO}_{2}, \mathrm{MoS}_{2}, \mathrm{WO}_{2}$, and $\mathrm{WS}_{2}$. 
that in the case of the disulfides. Therefore, the M-O $(\mathrm{M}=\mathrm{Mo}, \mathrm{W})$ bond is shorter than the M-S bond and cannot be broken easily. In other words, the transition metal dioxides exhibit higher bond stiffness at the interface than the disulfides.

Second, the interlayer interaction during the sliding course is taken into consideration. The static potential surfaces of the four solid lubricant materials at their equilibrium interlayer distances are shown in Fig. 4. All the materials exhibit a zigzag minimum energy sliding path. Furthermore, the transition metal dioxides exhibit identical interlayer friction potential barriers, and same is the case with the disulfides. However, the dioxides have a lower interlayer interaction than the disulfides. Therefore, according to the PrandtlTomlinson model and the view point of Dag and Ciraci [24], the higher bond stiffness at the interface and the lower interlayer interaction in the dioxides indicate their superlubricity. It is also necessary to investigate the intrinsic electron distribution of the single layer nanostructures. As shown in Fig. 5, the amplitude of the electron density of the transition metal disulfides is higher than that of the dioxides. This explains the lower equilibrium interlayer distance in the case of transition metal dioxides despite their more charged surface. Furthermore, the dioxides have a weaker electronic cloud density between the two neighboring oxygen atoms, which facilitates the oxygen atoms of the counterpart to go through. Therefore, the dioxides exhibit a better lubrication performance than the disulfides due to the sparse electron cloud distribution among the oxygen atoms at the interface. When the interlayer distance decreases to $0.5 \AA$, as shown in Fig. A1 of the Appendix, the lubrication performance can be arranged in the order $\mathrm{WS}_{2}>\mathrm{MoS}_{2}>\mathrm{MoO}_{2}>\mathrm{WO}_{2}$ by comparing the values of $\Delta V_{\max }\left(f_{\text {ext }}\right)$ and the potential barrier. However, the difference in the frictional potential barrier between the dioxides and the disulfides is only $0.1 \mathrm{eV}$. Furthermore, the stress in (a)

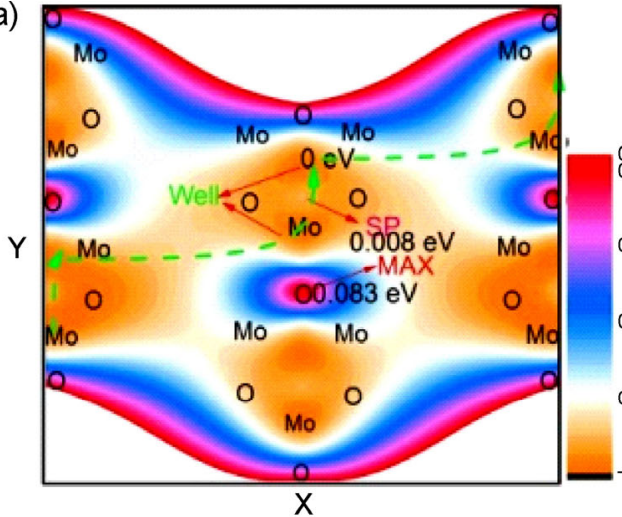

(c)

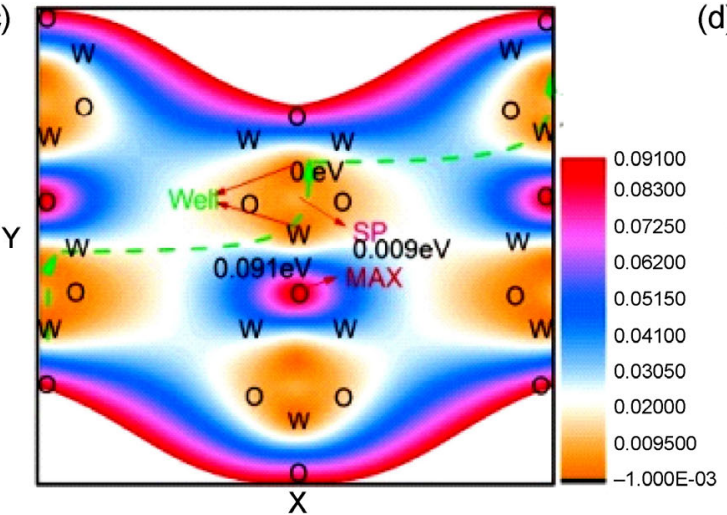

(b)

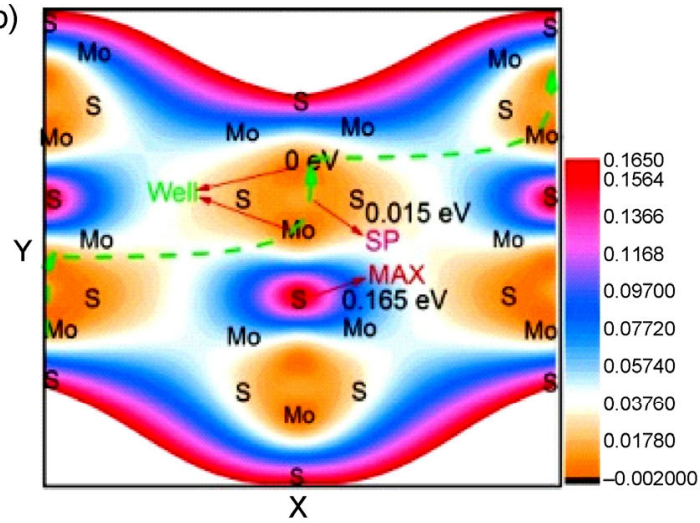

(d)

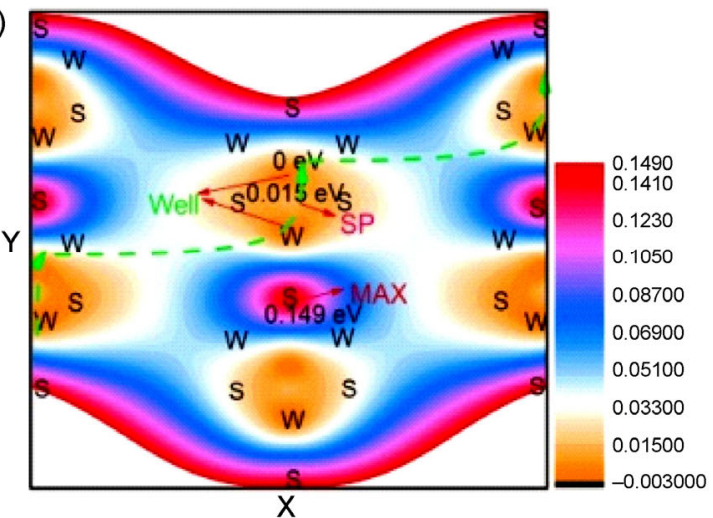

Fig. 4 Static potential surfaces of (a) $\mathrm{MoO}_{2}$, (b) $\mathrm{MoS}_{2}$, (c) $\mathrm{WO}_{2}$ and (d) $\mathrm{WS}_{2}$ at their equilibrium interlayer distance. Well indicates the minimum interaction energy, SP indicates the saddle point, and MAX indicates the maximum interaction energy. The curved arrows indicate the minimum energy sliding path. 


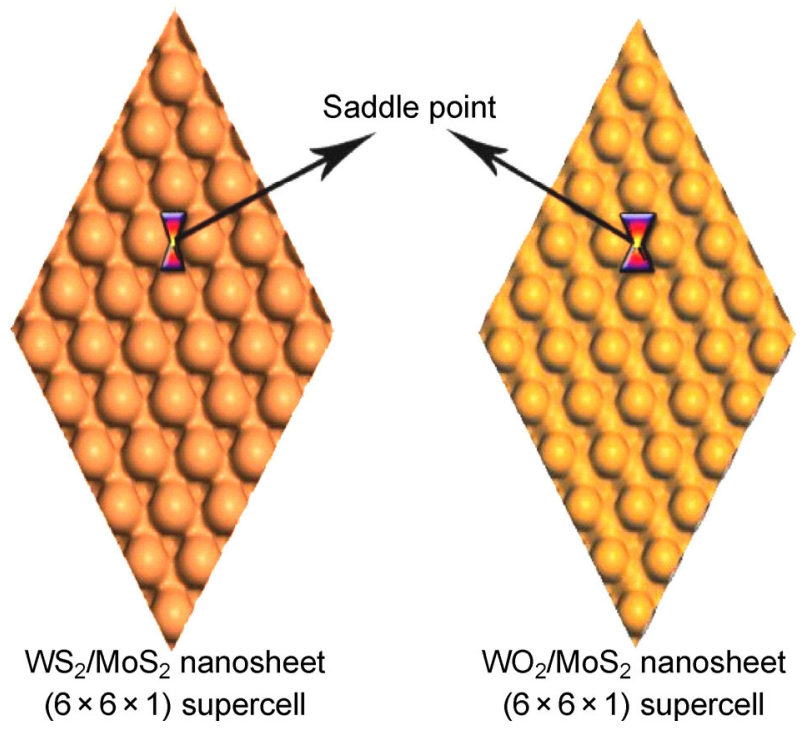

Fig. 5 Topography of the electron distribution of the single layer transition metal disulfide and dioxide nanostructures.

the $z$ direction as shown in Fig. A2 of the Appendix clearly indicates that such a small potential energy difference is obtained by almost doubling the normal load. Thus, at the same applied normal load, the dioxides exhibit a better lubrication performance.

Third, the molecular dynamic simulation results are analyzed. As shown in Fig. 6, during the whole stick-slip period, $\mathrm{MoS}_{2}$ and $\mathrm{WS}_{2}$ exhibit nearly the same value of friction work, and similar is the case with $\mathrm{MoO}_{2}$ and $\mathrm{WO}_{2}$. The lower positive friction work of the dioxides in the stick phase confirms the superlubricity of $\mathrm{MoO}_{2}$ and $\mathrm{WO}_{2}$.

Finally, the mechanical properties of the bulk solid lubricant materials are evaluated based on the first principles calculation. The results are summarized in Table 2. The transition metal dioxides exhibit nearly the same values of bulk modulus, Poisson's ratio and compressibility. The mechanical properties of the transition metal disulfides are also similar. However, the dioxides exhibit higher values compared to the disulfides. Therefore, we can conclude that the transitional metal dioxides demonstrate better load bearing capacities than the disulfides. Furthermore, the values of shear modulus and Young's modulus of the disulfides are comparable. It is interesting to note that $\mathrm{MoO}_{2}$ exhibits higher values of shear modulus and Young's modulus than those of $\mathrm{MoS}_{2}$ and $\mathrm{WS}_{2}$; however, these values are lower than those of $\mathrm{WO}_{2}$. The dioxides therefore exhibit higher mechanical stiffness than the disulfides making them difficult to deform. The higher load bearing capability and mechanical stiffness suggest that $\mathrm{MoO}_{2}$ and $\mathrm{WO}_{2}$ are better solid lubricants compared with their disulfides.
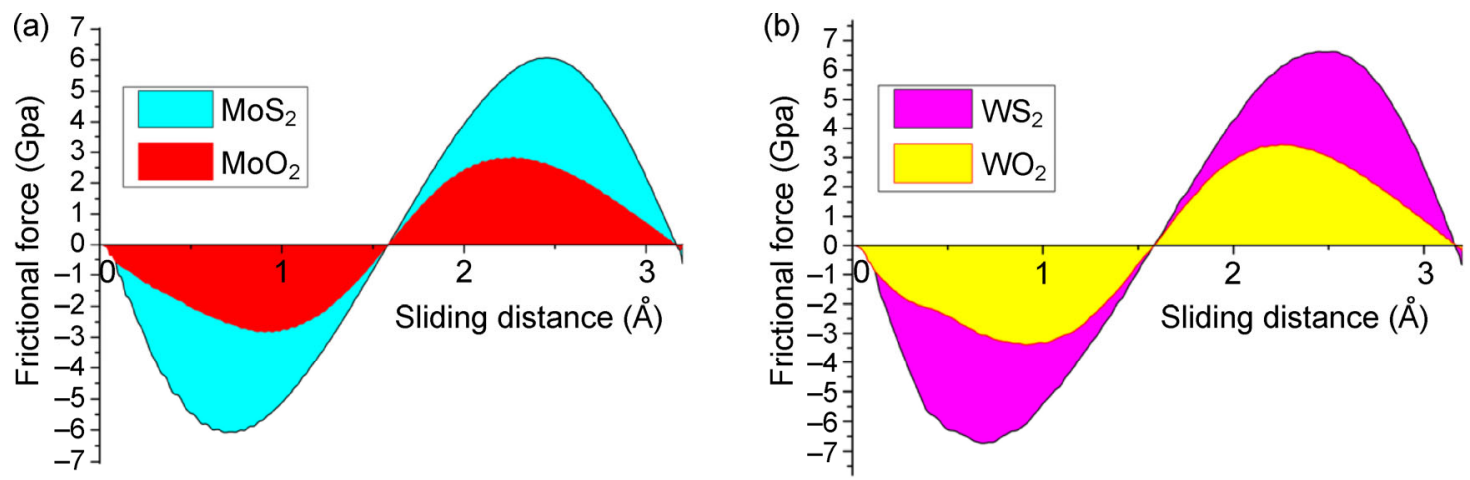

Fig. 6 Interlayer friction work done by (a) $\mathrm{MoS}_{2}$ and $\mathrm{MoO}_{2}$, and (b) $\mathrm{WS}_{2}$ and $\mathrm{WO}_{2}$, during friction in a commensurate condition.

Table 2 Mechanical properties of bulk $\mathrm{MoO}_{2}, \mathrm{MoS}_{2}, \mathrm{WO}_{2}$, and $\mathrm{WS}_{2}$.

\begin{tabular}{cccccc}
\hline System & Bulk modulus (Gpa) & Poisson ratio & Compressibility (1/Gpa) & Shear modulus (Gpa) & Young modulus (Gpa) \\
\hline $\mathrm{MoO}_{2}$ & 66.58 & 0.35 & 0.01502 & 103.6 & 471.41 \\
$\mathrm{MoS}_{2}$ & 58.06 & 0.23 & 0.01722 & 62.89 & 228.48 \\
$\mathrm{WO}_{2}$ & 64.38 & 0.33 & 0.01553 & 131.45 & 526.08 \\
$\mathrm{WS}_{2}$ & 56.26 & 0.19 & 0.01778 & 64.93 & 249.85 \\
\hline
\end{tabular}




\section{Conclusions}

The lubrication behaviors of lamellar dioxides and disulfides of molybdenum and tungsten were investigated theoretically and the results are summarized as follows.

(i) The topography of the electron density of the single layer nanostructures determined their sliding potential barrier. The sparser electron cloud distribution between the two neighbor oxygen atoms in the dioxides as compared to that of the disulfides facilitates the oxygen atoms of the counterpart to go through.

(ii) The higher bond strength at the interface, the lower interlayer interaction, and the lower positive friction work during the stick phase indicated the better lubricity of the dioxides than the disulfides.

(iii) The dioxides exhibit nearly similar lubrication performances, so do the disulfides.

(iv) The higher mechanical strength of the bulk dioxide materials indicated that they are better solid lubricants than the disulfides in vacuum.

\section{Acknowledgements}

This work is supported by the National Nature Science Foundation of China (Nos. 51522510 and 51675513), the "Top Hundred Talents" Program of Chinese Academy of Sciences and the National Key Basic Research and Development (973) Program of China (2013CB632300) for financial support.

Open Access: The articles published in this journal are distributed under the terms of the Creative Commons Attribution 4.0 International License (http:// creativecommons.org/licenses/by/4.0/), which permits unrestricted use, distribution, and reproduction in any medium, provided you give appropriate credit to the original author(s) and the source, provide a link to the Creative Commons license, and indicate if changes were made.

\section{Appendix}
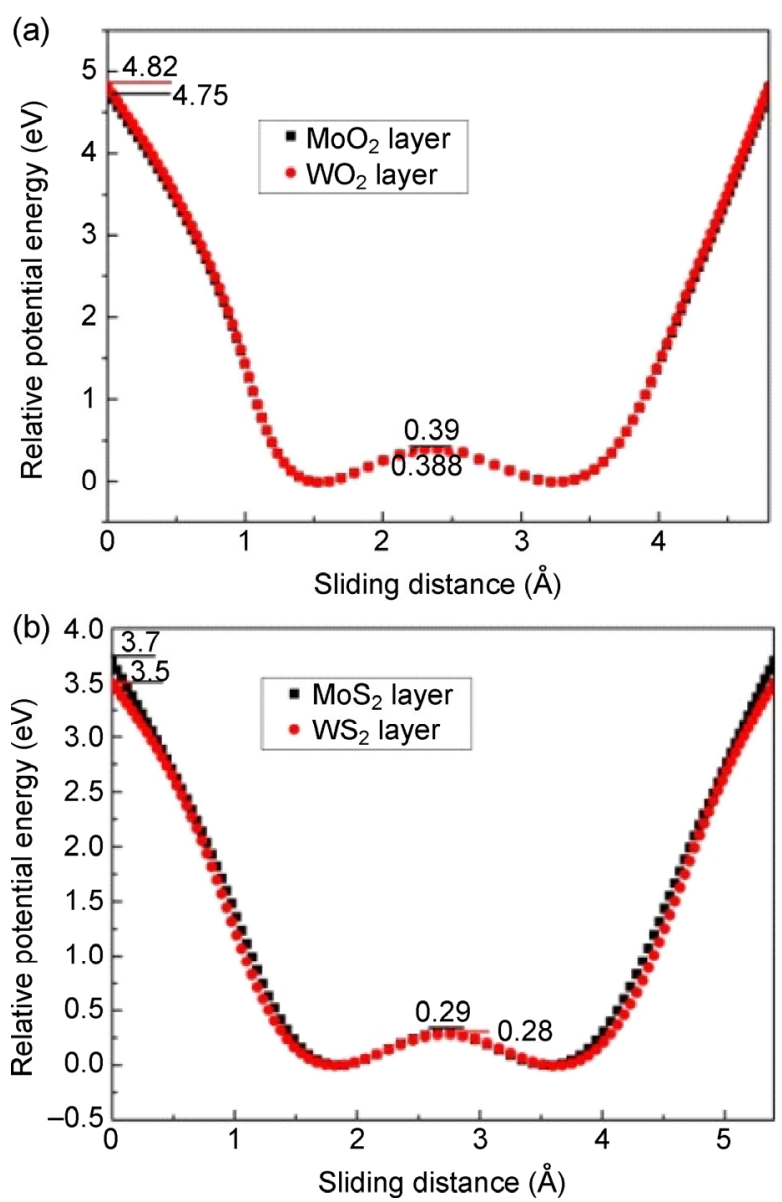

Fig. A1 (a) Relative sliding potential energy curves of transition metal dioxides when interlayer distance decreases $0.5 \AA$. (b) Relative sliding potential energy curves of transition metal disulfides when interlayer distance decreases $0.5 \AA$.

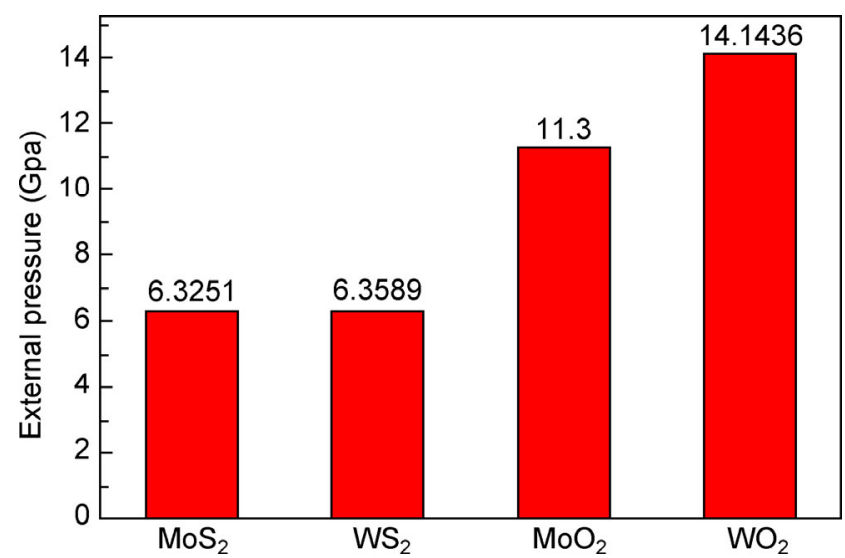

Fig. A2 The normal load is necessary to compress inter-layer distance down by $0.5 \AA$. 


\section{References}

[1] Grossiord C, Varlot K J, Martin M, Le Mogne T, Esnouf $\mathrm{C}$, Inoue $\mathrm{K} . \mathrm{MoS}_{2}$ single sheet lubrication by molybdenum dithiocarbamate. Tribol Int 31: 737-743 (1998)

[2] Martin J M, Grossiord C, Le Mogne T, Igarashi J. Transfer films and friction under boundary lubrication. Wear $\mathbf{2 4 5}$ : 107-115 (2000)

[3] De Barros Bouchet M I, Martin J M, Le Mogne T, Bilas P, Vacher B, Yamada Y. Mechanisms of $\mathrm{MoS}_{2}$ formation by MoDTC in presence of ZnDTP: Effect of oxidative degradation. Wear 258: 16430-16450 (2005)

[4] Stefanov M, Enyashin A N, Heine T, Seifert G. Nanolubrication: How do $\mathrm{MoS}_{2}$-based nanostructures lubricate? J Phys Chem C 112: 17764-17767 (2008)

[5] Onodera T, Morita Y, Suzuki A, Koyama M, Tsuboi H, Hatakeyama N, Endou A, Takaba H, Kubo M, Fabrice D, Minfray C, Joly-Pottuz L, Martin J M., Miyamoto A. A computational chemistry study on friction of h-MoS 2 . Part I. Mechanism of single sheet lubrication. J Phys Chem B 113: 16526-16536 (2009)

[6] Onodera T, Morita Y, Nagumo R, Miura R, Suzuki A, Tsuboi H, Hatakeyama N, Endou A, Takaba H, Dassenoy F, Minfray C, Joly-Pottuz L, Kubo M, Martin J M, Miyamoto A. A computational chemistry study on friction of h-MoS 2 . Part II. Friction anisotropy. J Phys Chem B 114: 1583215838 (2010)

[7] Wang C Q, Li H S, Zhang Y S, Sun Q, Jia Y. Effect of strain on atomic-scale friction in layered $\mathrm{MoS}_{2}$. Tribol Int 77: 211-217(2014)

[8] Levita G, Cavaleiro A, Molinari E, Polcar T, Righi MC. Sliding properties of $\mathrm{MoS}_{2}$ layers: Load and interlayer orientation effects. J Phys Chem C 118: 13809-13816 (2014)

[9] Cahangirov S, Ataca C, Topsakal M, Sahin H, Ciraci S. Frictional figures of merit for single layered nanostructures. Phys Rev Lett 108: 126103(2012)

[10] Prandtl L, Angew Z. A conceptual model to the kinetic theory of solid bodies. Math Mech 858: 1-19 (1928)

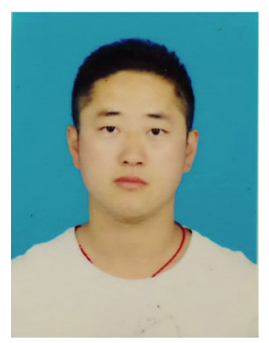

Jingyan NIAN. He received his master degree in physical chemistry in 2012 from Northwest Normal University, Lanzhou, China. During his undergraduate, he joined the
[11] Tomlinson G A. A molecular theory of friction. Philos Mag J Sci 7: 905-939 (1929)

[12] Mak K F, Lee C, Hone J, Shan J, Heinz T F, Atomically thin $\mathrm{MoS}_{2}$ : A new direct-gap semiconductor. Phys Rev Lett 105: 136805 (2010)

[13] Wang Z, Zhao K, Li H, Liu Z, Shi Z, Lu J, Suenaga K, Joung S K, Okazaki T, Jin Z, Gu Z. Gao Z, Iijima S. Ultranarrow $\mathrm{WS}_{2}$ nanoribbons encapsulated in carbon nanotubes. J Mater Chem 21: 171-180 (2011)

[14] Ataca C, Şahin H, Ciraci S. Stable single-layer MX 2 transitionmetal oxides and dichalcogenides in a honeycomb-like structure. J Phys Chem C 116: 8983-8999 (2012)

[15] Winkler B, Pickard C J, Segall M D, Milman V. Density functional study of charge ordering in $\mathrm{Cs}_{2} \mathrm{Au}(\mathrm{I}) \mathrm{Au}(\mathrm{III}) \mathrm{Cl}_{6}$ under pressure. Phys Rev B 63: 14103 (2001)

[16] Wu Z, Cohen R E. More accurate generalized gradient approximation for solids. Phys Rev B 73: 235116 (2006)

[17] Ceperley D M, Alder B J. Ground state of the electron gas by a stochastic method. Phys Rev Lett 45: 566-569 (1980)

[18] Perdew J P, Zunger A. Self-interaction correction to density-functional approximations for many-electron systems. Phys Rev B 23: 5048-5079 (1981)

[19] Zhong W, Tomanek D. First-principles theory of atomicscale friction. Phys Rev Lett 64: 3054-3057 (1990)

[20] Girifalco L A, Hodak M. Van der Waals binding energies in graphitic structures. Phys Rev B 65: 125404 (2002)

[21] Grimme S. Semiempirical GGA-type density functional constructed with a long-range dispersion correction. J Comput Chem 27: 1787 (2006)

[22] Wu Z J, Zhao E J, Xiang H P, Hao X F, Liu X J, Meng J. Crystal structures and elastic properties of superhard $\mathrm{IrN}_{2}$ and $\mathrm{IrN}_{3}$ from first principles. Phy Rev B 76: 054115 (2007)

[23] Rappe A K, Colwell K S, Casewit C J. Application of a universal force field to metal complexes. Inorg Chem 32: 3438-3450 (1993)

[24] Dag S, Ciraci S. Atomic scale study of superlow friction between hydrogenated diamond surfaces. Phys Rev B 70: 241401 (2004)

State Key Laboratory of Solid Lubrication at Lanzhou Institute of Chemical Physics, Chinese Academy of Sciences. His current position is an associate researcher. His research area covers the atomic-scale friction, superlubricity, and space lubrication. 


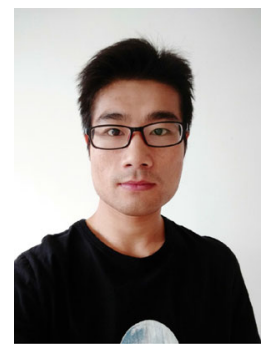

Liwei CHEN. He joined Prof. Zhiguang GUO's biomimetic materials of tribology (BMT) group at University of Hubei in 2014 in

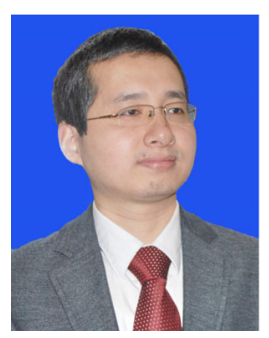

Zhiguang GUO. He received his Ph.D. degree from Lanzhou Institute of Chemical Physics (LICP), Chinese Academy of Sciences (CAS) in 2007. During Feb. 2009 to Feb. 2010, he worked in Department of Physics,

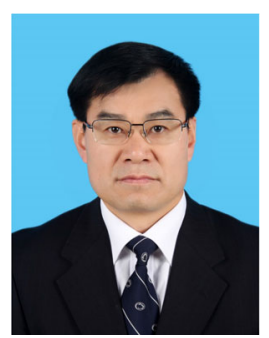

Weimin LIU. He received his Ph.D. degree with major of Lubricating Materials and Tribology from Lanzhou Institute of Chemical Physics (LICP), Chinese Academy of Sciences in 1990. In 2013, he was pursuing his Ph.D. degree. His current scientific interests are devoted to designing and fabricating superamphiphobic materials for the separation of oil/water mixtures.
University of Oxford, UK, as a visiting scholar. Now he is a full professor in LICP financed by "Top Hundred Talents" program of CAS. Till now, he has published more than 130 papers about the interfaces of materials. elected as the fellow of Chinese Academy of Sciences, and now, he is the director of the state key lab of solid lubrication. Up to now, he has published more than 500 papers and his research interests mainly focus on space lubrication and high performance lubricants. 